\title{
Analysis of spiral arms using anisotropic wavelets: gas, dust and magnetic fields in M 51
}

\author{
I. Patrikeev ${ }^{1, \star}$, A. Fletcher ${ }^{2,3}$, R. Stepanov ${ }^{1}$, R. Beck ${ }^{2}$, E. M. Berkhuijsen ${ }^{2}$, P. Frick ${ }^{1}$, and C. Horellou ${ }^{4}$ \\ 1 Institute of Continuous Media Mechanics, Korolyov str. 1, 614061 Perm, Russia \\ 2 Max-Planck-Institut für Radioastronomie, Auf dem Hügel 69, 53121 Bonn, Germany \\ 3 School of Mathematics and Statistics, University of Newcastle, Newcastle upon Tyne NE1 7RU, UK \\ e-mail: andrew.fletcher@ncl.ac.uk \\ ${ }^{4}$ Onsala Space Observatory, Chalmers University of Technology, 43992 Onsala, Sweden
}

Received 17 March 2006 / Accepted 1 August 2006

ABSTRACT

\begin{abstract}
Context. The origin of the spiral pattern of magnetic fields in disc galaxies is an open question.
Aims. Comparison of the regular magnetic field orientation with the gaseous spiral arm pitch angles can tell us whether spiral shock compression is responsible for the magnetic spirals. We also wish to see whether the ridges of different components of the ISM show the large-scale, systematic shifts expected from density wave theory.

Methods. We have developed a technique of isolating elongated structures in galactic images, such as spiral arms, using anisotropic wavelets and apply this to maps of the CO, infrared and radio continuum emission of the grand-design spiral galaxy M 51.

Results. Systematic shifts between the ridges of CO, infrared and radio continuum emission that are several kpc long are identified, as well as large variations in pitch angle along spiral arms, of a few tens of degrees. We find two types of arms of polarized radio emission: one has a ridge close to the ridge of $\mathrm{CO}$, with similar pitch angles for the $\mathrm{CO}$ and polarization spirals and the regular magnetic field; the other does not always coincide with the $\mathrm{CO}$ arm and its pitch angle differs from the orientation of its regular magnetic field.

Conclusions. The offsets between ridges of regular magnetic field, dense gas and warm dust are compatible with the sequence expected from spiral density wave triggered star formation, with a delay of a few tens of millions of years between gas entering the shock and the formation of giant molecular clouds and a similar interval between the formation of the clouds and the emergence of young star clusters. At the position of the $\mathrm{CO}$ arms the orientation of the regular magnetic field is the same as the pitch angle of the spiral arm, but away from the gaseous arms the orientation of the regular field varies significantly. Spiral shock compression can explain the generation of one type of arm of strong polarized radio emission but a different mechanism is probably responsible for a second type of polarization arm.
\end{abstract}

Key words. galaxies: spiral - galaxies: magnetic fields - galaxies: ISM - galaxies: individual: M 51 - methods: data analysis

\section{Introduction}

Disc galaxies often display spiral patterns in their distributions of stars, gas, dust and magnetic fields. Spiral structure is present in both the distribution of the radio synchrotron intensity, and the orientation of the kpc-scale regular field, as measured by the $\boldsymbol{B}$-vectors of radio polarization (e.g. Beck 2005). Comparing the location and orientation of the spiral patterns in different components of the interstellar medium (ISM) can provide important information on the astrophysical connections between interstellar gas, dust and magnetic fields in galaxies.

Usually the prominent arms in disc galaxies are treated as having a logarithmic spiral pattern, where the pitch angle of the arm is constant along its length. However, Kennicutt (1981) found that the logarithmic spiral form is no better a mathematical description of real spirals than hyperbolic forms, and that "... small scale distortions preclude the possibility of any universal shape for galactic spirals". Deviations from the logarithmic spiral may be caused by local disturbances or arise from systematic global effects, such as the gravitational force of a nearby

^ Current address: 301 University Blvd., UTMB, Galveston, TX, USA, 77550-0456; e-mail: igpatrik@utmb.edu galaxy or the presence of more than one spiral density wave (Elmegreen et al. 1989). If the regular magnetic field spirals are being aligned with the gaseous spiral arms by shock compression, the magnetic field orientation should be closely related to the local spiral arm pitch angle. In order to quantify how well the different spirals are aligned a robust method of measuring local pitch angles is required.

M 51 is probably perturbed by a recent encounter with its companion galaxy NGC 5195. Such interactions often result in increased star formation rates, either localised or global, as tidal forces and spiral density waves compress the interstellar medium. The two spiral arms of M51 can be traced through more than $360^{\circ}$ in azimuthal angle in numerous wavebands and several authors have investigated their structure. Elmegreen et al. (1989) de-projected images taken in the optical $B$ and $I$ bands into the $\log$ (radius)-azimuth plane and used the observed amplitude variations along the arms to locate resonances of spiral modes. They concluded that M 51 contains an inner and an outer system of spiral arms with a conjunction of resonances at $2^{\prime}-3^{\prime}$ galacto-centric radius, triggered by the companion. Measurements of the pitch angles of the main dust lanes by Howard \& Byrd (1990) and of the variation in star formation efficiency along the arms by Knapen et al. (1992) confirmed this 
double spiral structure. It is clear from a cursory visual inspection that even the well defined - grand design - spiral arms of M 51 have many variations in pitch angle along their length.

Radial shifts between the locations of the crest of the spiral arms in different constituents are expected from density wave theory. Petit et al. (1996) observed a 300-400 pc shift between $\mathrm{H} \alpha$ and UV arms. Rand \& Kulkarni (1990), Scoville et al. (2001), and Tosaki et al. (2002) noted that the $\mathrm{H} \alpha$ arms are located outside the CO arms. Henry et al. (2003) found that the Paschen- $\alpha$ line emission is offset from the $\mathrm{CO}$ emission along part of the arms. All these shifts refer to the inner spiral system and are expected for arms triggered by density waves. Tilanus et al. (1988) found that thermal radio continuum and $\mathrm{H} \alpha$ arms are significantly shifted outwards from the dust lanes, whereas the non-thermal radio continuum arms occur just inside the dust lanes.

Most of the above analyses were made by overlaying the contours of one image on a grey-scale map of the other or by making azimuthal cuts through the data at different radii. Both methods, while showing the most significant local displacements in the position of the arms, make it difficult to see the extent along the arm of any systematic shift. In the present paper we describe an objective method, using wavelet analysis, to determine the location of the spiral arm ridges, and measure their local pitch angles, along the entire length of an arm. We apply this method to images of the total and polarized radio continuum emission at $\lambda 6 \mathrm{~cm}$ (Fletcher et al. in preparation), the dust emission at $15 \mu \mathrm{m}$ (Sauvage et al. 1996) and the $\mathrm{CO}(1-0)$ line emission (Helfer et al. 2003).

In Sect. 2 we describe the anisotropic wavelet transform and how we use this method to identify the location of a spiral arm and to measure its localised pitch angles. The observational data we use are briefly discussed in Sect. 3 and the results of applying the anisotropic wavelet method to the data are given in Sect. 4. The astrophysical implications of the results are discussed in Sect. 5.

\section{The method}

In image analysis, wavelet based methods allow the isolation of features, such as spiral arms, and the decomposition of a map into a hierarchy of structures on different scales. Wavelets are a tool for data analysis based on self-similar functions which are well localised both in the physical and frequency domains. Using one-dimensional isotropic wavelets Frick et al. (2000) identified systematic shifts between the magnetic and optical spiral arms in NGC 6946 and using two-dimensional isotropic wavelets Frick et al. (2001) investigated the scale-by-scale correlations between maps of the same galaxy in different spectral ranges. In this paper a new method, using a two-dimensional anisotropic wavelet is presented.

The wavelet transform can be considered as a generalization of the Fourier transform. The classical Fourier transform is based on harmonic functions. The generalized Fourier transform allows using non-harmonic orthogonal functions as a basis (e.g. a Walch set of discrete, piecewise-constant functions). The shorttime Fourier transform and the Gabor transform use oscillatory basis functions with local support. The wavelet transform also uses oscillatory functions, but in contrast to the classical Fourier transform these functions decay rapidly toward infinity and all functions in the wavelet basis are self-similar (the main distinction between the wavelet transform and the Gabor transform). One-dimensional and isotropic multi-dimensional wavelet transforms are based on the space-scale decomposition of the signal (in other words, the family of wavelets has two parameters, governing the location and the size of the basis function). Using the continuous isotropic wavelet transform, a 2D image is decomposed into a 3D cube of wavelet coefficients. Cross-sections of the cube are slices which contain the image details at a fixed scale. As a result, the wavelet transform conserves the local properties of the image at all scales. If required, the original image can be reconstructed from the cube by summing over all scales (this procedure is called the inverse wavelet transformation).

An anisotropic wavelet transform is the convolution of the image with a set of wavelets having different locations, sizes and orientations. Such a family of basis functions is generated by translations, dilations and rotations of the basic wavelet. Applying the two-dimensional anisotropic wavelet transform to an image generates a four-dimensional data set, which is a spacescale-orientation decomposition. Fixing the space and scale parameters - based on some objective criteria - enables one to track the orientation of an elongated structure (Antoine 1993). An extended description of the continuous wavelet transform can be found, for example, in Holschneider (1995) and Torresani (1995).

\subsection{The Texan Hat function}

The ideal anisotropic wavelet for astronomical image processing should combine high angular sensitivity with a simple computational formula; the latter requirement is due to the time required to calculate the wavelet transform of a high resolution image. In this work we use a specially designed ad hoc anisotropic wavelet. Its formula is very simple and the wavelet transformation can be calculated efficiently. We do not use the Cauchy wavelet (Antoine 1993), which provides the best angular sensitivity, due to its more complicated formula.

We introduce our wavelet by starting with the Mexican Hat function $(\mathrm{MH})$, one of the most commonly used wavelets. In the $1 \mathrm{D}$ case, the $\mathrm{MH}$ is described by $\psi(x)=\left(1-x^{2}\right) \exp \left(-x^{2} / 2\right)$. The 1D MH can be extended into 2D in one of two ways: (i) constructing an isotropic (axisymmetric) function by rotation of the 1D MH, (ii) translation of the 1D MH along an axis but restricting it within a (e.g. Gaussian-shaped) window in this direction, to obtain an anisotropic function. We call the anisotropic wavelet constructed using the latter method the Texan Hat function (TH) and it can be represented by

$\psi(x, y, a)=\left[1-\left(\frac{y}{a}\right)^{2}\right] \exp \left(-\frac{x^{2}+y^{2}}{2 a^{2}}\right)$

where $a$ is the scale parameter by which the wavelet is dilated. In the form of Eq. (1) the TH is sensitive to structures elongated along the $x$-axis. This means that if an image is convolved with the TH, extended objects parallel to the $x$-axis will be amplified. Substitution of $(x \cos \varphi+y \sin \varphi)$ for $x$ and $(y \cos \varphi-x \sin \varphi)$ for $y$ in Eq. (1) makes the TH sensitive to its orientation $\varphi$ measured from the $x$-axis, since this substitution is equivalent to a rotation of the axes by $\varphi$. A complete set of basis functions of different sizes and orientations is obtained by dilation and rotation of the basic wavelet. Note that since the $\mathrm{TH}$ is a symmetric function with respect to rotation by $180^{\circ}$ it is sensitive to the orientation of an elongated structure, not to its direction:

$\psi_{\varphi}(x, y)=\psi_{\varphi+180^{\circ}}(x, y)$

Another, more formal, way to design the TH is as follows. The 1D MH can be defined as the second derivative of the 



Fig. 1. Analysis of an artificial spiral image with the Texan Hat function. a) Two arm logarithmic spiral with a central exponential disc and randomly distributed, slightly smoothed, point sources. The pitch angle of the first (unbroken) arm is $20^{\circ}$; the pitch angle of the second (broken) arm is $15^{\circ}$ at small radii and $25^{\circ}$ at large radii. b) Map of the maximum wavelet coefficients at a fixed scale that is close to the average arm width.

Gaussian function. Similarly, the TH is defined as the second partial derivative of the 2D Gaussian function:

$\psi(x, y)=-\frac{\partial^{2}}{\partial y^{2}} \exp \left(-\frac{x^{2}+y^{2}}{2}\right)$

Equation (3) can be parametrized for variation in its size $a$ by substituting $x$ with $x / a$ and $y$ with $y / a$ (i.e. a dilation of the axes). This approach is useful for determining the properties of the $\mathrm{TH}$ in the Fourier domain. For example, one can easily estimate the Fourier transform of the TH by using the Fourier transform of the Gaussian function and the theorem of derivatives in the Fourier domain. Furthermore the definition of the wavelet as a derivative is not only valid for the $2 \mathrm{D}$ case, but also for higher dimensions.

The anisotropy of the TH can be successfully exploited for an analysis of the orientations of galactic spiral arms. A brief overview of the method was first presented in Patrikeev et al. (2005). A detailed description of the technique and an illustrative application to an artificial spiral image are given in the next section.

\subsection{Measurement of spiral arm position and pitch angles}

It is obvious that for an isotropic structure (e.g. a round-shaped object, or randomly distributed set of spots, etc.) an orientation cannot be defined. In this work we use the TH wavelet to trace the ridge of a spiral arm and to measure its local pitch angles. To illustrate how the TH can determine the position and pitch angle of a spiral arm, we use the artificial image presented in Fig. 1a. The test image consists of: (1) two logarithmic spiral arms with Gaussian cross-sectional profiles, the first has a constant pitch angle of $20^{\circ}$ and the second, broken, arm has a pitch angle of $15^{\circ}$ at small radii and $25^{\circ}$ at large radii; (2) an exponential disc that is about $70 \%$ of the arm intensity in the central region; (3) fifty bright point sources, slightly smoothed with a Gaussian kernel, distributed randomly over the image.

The test image was convolved with a TH at a fixed scaling parameter $a$ close to the average spiral width and a rotation parameter $\varphi$ ranging from $0^{\circ}$ to $180^{\circ}$ in steps of $1^{\circ}$. From the convolution we obtained a stack of maps of wavelet coefficients; each map represents a specific value of $\varphi$ and has the same size as the

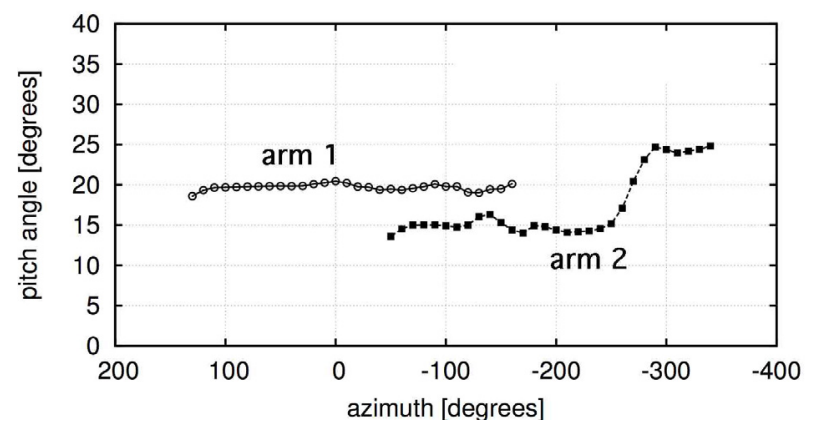

Fig. 2. Azimuthal variation of pitch angle determined from the anisotropic wavelet transform of the spiral arms of Fig. 1a. Pitch angles are measured at fixed azimuthal increments along the ridges of the arms. The azimuthal angle is measured counter-clockwise from the top of the image.

test image. Figure 1b shows the map of maximum wavelet coefficients, obtained by taking the largest value through the stack at each position. The central exponential background is invisible because the wavelet coefficients of such a structure are very low. The point sources are converted to faint spots; their wavelet coefficients have no pronounced maximum since a round source has no orientation. In contrast to the non-oriented structures, the two-fold spiral becomes more emphatic, with large wavelet coefficients.

We define the location of the ridge of a spiral arm as the position where the maximum wavelet coefficient is found. The local pitch angle is defined as the TH orientation corresponding to the maximum wavelet coefficient. Figure 2 shows the measured azimuthal variation of pitch angle at the ridge of the spirals. The first arm has a pitch angle close to the expected value of $20^{\circ}$. The pitch angle of the second arm changes from $15^{\circ}$ to $25^{\circ}$ (near the azimuth about $-270^{\circ}$ ) remaining constant before and after the break. Small deviations of the pitch angle are caused by the influence of bright sources located in the vicinity of the spirals.

The analysis of the test image demonstrates the capability of the proposed method to determine the pitch angle of the spiral and to resolve the difference of $5^{\circ}$ despite the exponential background and small-scale bright sources.

\section{The data}

M 51 is a well observed galaxy and data in many spectral ranges are available. For our analysis we use four maps: $\mathrm{CO}(1-0)$ line emission of molecular gas, total and linearly polarized radio continuum emission at $\lambda 6.2 \mathrm{~cm}$, and mid-infrared dust emission at $15 \mu \mathrm{m}$.

The $\mathrm{CO}(1-0)$ line emission at $\lambda 2.6 \mathrm{~mm}$ was observed with the BIMA interferometer and corrected for missing spacings with single dish observations from the NRAO $12 \mathrm{~m}$ dish on Kitt Peak (Helfer et al. 2003). CO emission is the best available tracer of the molecular gas in M 51.

It is often assumed that dust lanes are the best tracers of large scale spiral arm shocks, although recent Hubble Space Telescope images show a plethora of dust-absorption spurs and branches attached to the M 51 spiral arms that clearly require a more subtle interpretation. The recent $850 \mu \mathrm{m}$ map of M 51 at $15^{\prime \prime}$ resolution - twice the resolution of the data used in this paper by Meijerink et al. (2005) shows that most of the (cold) dust in M 51 originates from an exponential disc, rather than from narrow spiral arms associated with the dust-absorption lanes seen in 
the optical bands. We note that at high resolution the strongest $\mathrm{CO}(1-0)$ emission appears to closely follow the main spiral dust lanes in the inner $r \lesssim 4 \mathrm{kpc}$ (Aalto et al. 1999).

The $\lambda 6.2 \mathrm{~cm}$ total and linearly polarized radio continuum emission was observed with the VLA ${ }^{1}$ and corrected for missing short baselines using maps obtained with the Effelsberg ${ }^{2}$ telescope (Fletcher et al. in preparation). The total emission is a mixture of synchrotron radiation of cosmic-ray electrons spiralling in interstellar magnetic fields and bremsstrahlung emission from thermal electrons. The relative fractions of synchrotron and thermal emission vary with position; at the location of $\mathrm{H}$ II regions in the spiral arms the emission is up to $50 \%$ thermal but in the inter-arm regions this fraction falls to $\leq 20 \%$. Hence, the total radio intensity $\mathrm{I} 6$ is mostly a measure of the total strength of the magnetic field and the cosmic ray electron density. The polarized emission PI6 is purely non-thermal, and its intensity is a measure of the strength of the regularly oriented magnetic field ${ }^{3}$. The polarization $\boldsymbol{B}$-vectors were corrected for Faraday rotation using new $\lambda 3.5 \mathrm{~cm}$ data (Fletcher et al. in preparation) and show the orientation of the regular magnetic field. The similarity of the polarized intensity maps at $\lambda \lambda 3.5,6.2 \mathrm{~cm}$ (apart from generally reduced intensities at $\lambda 3.5 \mathrm{~cm}$ in line with the non-thermal spectral index) and the low Faraday rotation measures mean that Faraday depolarization does not significantly effect the PI6 map.

The $15 \mu \mathrm{m}$ dust emission (denoted ISO throughout this paper) was observed with ISOCAM (Sauvage et al. 1996) and is a combination of thermal continuum emission from dust particles and line emission from PAH molecules.

All maps were smoothed to $8^{\prime \prime}$ resolution, equivalent to $\simeq 400 \mathrm{pc}$ at the assumed distance of M 51 (9.7 Mpc, Sandage \& Tammann 1974). The maps were rectified to a face-on orientation using an assumed inclination of $20^{\circ}$ (where $0^{\circ}$ is face-on) and a position angle of $-10^{\circ}$ for the orientation of the major axis (Tully 1974). Figure 3 shows the face-on maps at $8^{\prime \prime}$ resolution.

If the de-projection to face-on orientation uses incorrect parameters and the spiral arms have a simple mathematical description, such as logarithmic spirals, sine-wave like oscillations will appear in $\log$ (radius)-azimuth plots of the ridges (see Fig. 2 of Kennicutt 1981). For $r<6 \mathrm{kpc}$ we do not find any such oscillations in either arm, but for $r>6 \mathrm{kpc}$ a series of 3 peaks and troughs is present in the I6 and ISO of arm 1 (Fig. 6). The pattern may indicate a distortion of the disc in this region of M 51, rather than intrinsic variability in the spiral pattern. Without detailed information on the velocity field we cannot investigate this possibility; however, whatever the cause of the oscillations, the relative positions of the I6 and ISO arms will be the same.

\section{Results}

\subsection{The anisotropic wavelet transform}

Figure 4 shows the maximum wavelet coefficients for the four maps in Fig. 3. The scale parameter $a$ of the wavelet was fixed

1 The VLA is operated by the NRAO. The NRAO is a facility of the National Science Foundation operated under co-operative agreement by Associated Universities, Inc.

2 The Effelsberg telescope is operated by the Max-Planck-Institut für Radioastronomie on behalf of the Max-Planck-Gesellschaft.

3 A note on terminology is required. In this paper we use the term "regular magnetic field" to refer to the magnetic field that gives rise to the observed polarized synchrotron emission. Polarized emission can be produced by an anisotropic random magnetic field as well as a coherent mean field; in this work we ignore the distinction except for a brief discussion in Sect. 5. to the approximate width of the spiral arms; tests showed that the results are insensitive to the aspect ratio of the wavelet in the range $0.5-2$ times the chosen aspect ratio. The anisotropic wavelet transform clearly picks out the elongated arms in the images. In addition, smaller scale structures such as spurs roughly perpendicular to the arms are identified. In the PI6 map the low signal to noise ratio (relative to the other maps) and the patchiness of the diffuse emission results in a web of structures showing up in Fig. 4d, however extended arms of polarized emission are also evident.

The location of the wavelet coefficient maxima at a given radius are interpreted as the position of the spiral arm ridge. To ensure an even spacing of measured positions we select an initial point that clearly lies on a spiral arm and then choose the location with the maximum wavelet coefficient that is a fixed increment in azimuth away from the initial position (2 degrees) within an arc of fixed opening angle. The anisotropic wavelet will pick out spurs and ridges that are connected to the spiral arms (Fig. 4) but by selecting the maximum wavelet coefficient within an arc we attempt to pick out the continuous structure of the spiral arm. The pitch angle of the arm is given by the orientation of the anisotropic wavelet that generates the maximum wavelet coefficient at each of these positions.

Uncertainties in the measured pitch angle are caused by (i) the form of the real structures (they can be curved, asymmetric, having varying width etc.) and (ii) by instrumental noise in the map. In our approach structures on scales smaller than the scale of the arms - such as discrete bright clouds, spurs and breaks - can be considered as noise. To calculate the errors in our measured pitch angles we used a Monte-Carlo technique. We define all structures on scales less than the approximate width of the spiral arms of $1 \mathrm{kpc}$ as belonging to the noise. We carry out large-scale wavelet filtration of the image, keeping only scales $a<1 \mathrm{kpc}$ and consider this to be a map of the noise. Then we reanalyse the original map overlaid with the noise map shifted (the noise map can be laterally shifted, rotated or reflected) by an arbitrary amount and calculate the pitch angles again. The process is repeated using several arbitrarily shifted noise maps to obtain the standard deviation for the pitch angle of the arms.

\subsection{The location of the spiral arms}

Figure 5 shows the location of the spiral arm ridges in M 51 derived from the anisotropic wavelet transform (Fig. 4) of the total and polarized $\lambda 6 \mathrm{~cm}, \mathrm{CO}$ and ISO $15 \mu \mathrm{m}$ maps. An alternative view of the same data - in $\log (r)$-azimuth co-ordinates - is shown in Fig. 6.

The small-amplitude "wiggles" in the plotted ridge lines are due to the fixed number of positions where the ridge position was measured and do not translate into pitch angle fluctuations.

There are three systematic offsets between the spiral arms traced in different wavebands; the most obvious offset is that of the polarized emission, but since this is the most complicated to discuss we will leave it to last.

First, the CO spiral arms are consistently situated on the concave side of the ISO $15 \mu \mathrm{m}$ arms. The shift between the two ridges is typically $\sim 100 \mathrm{pc}$, increasing to around $600 \mathrm{pc}$ in the northern part of arm 2 . This offset fits neatly with the chronology of the conversion of gas into stars in the large-scale shock model of spiral arms (Roberts 1969; Shu et al. 1972; Tosa 1973). The CO emission traces molecular gas clouds, whose formation is triggered by a shock on the upstream side of the spiral arm (the concave side within the co-rotation radius where the gas rotates faster than the spiral pattern). After dense cores in these clouds 

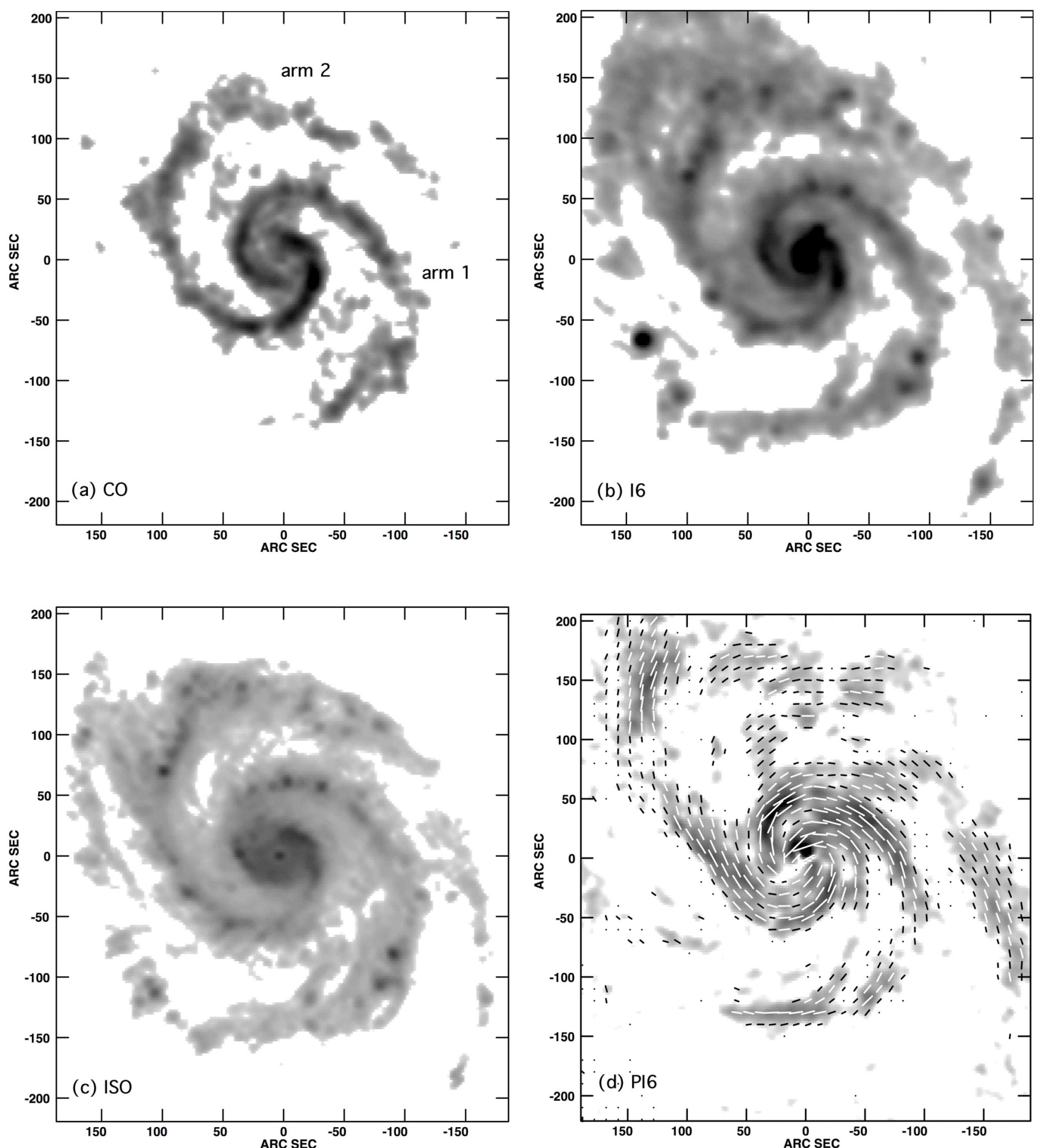

Fig. 3. The maps analysed in this paper. a) $\mathrm{CO}(1-0)$ emission (Helfer et al. 2003). b) Total radio emission at $\lambda 6 \mathrm{~cm}$ (Fletcher et al. in preparation). c) Infrared emission at $15 \mu \mathrm{m}$ (Sauvage et al. 1996). d) Linearly polarized $\lambda 6 \mathrm{~cm}$ radio emission (Fletcher et al. in preparation), with $\boldsymbol{B}$-vectors showing the orientation of the regular magnetic field (corrected for Faraday rotation). A grey-scale proportional to the square root of the intensity has been used to emphasise the spiral structures. Arms 1 and 2 are indicated on the CO map. The resolution is $8^{\prime \prime}$ in all maps and all have been rectified to a face-on orientation. The co-ordinate system is in seconds of arc relative to the galaxy centre $\left(\mathrm{RA}\right.$, Dec $(2000): 13^{\mathrm{h}} 27^{\mathrm{m}} 46^{\mathrm{s}}$, $\left.+47^{\circ} 27^{\prime} 10^{\prime \prime}\right)$.

have collapsed, stars form downstream of the position where the clouds formed. The ISO $15 \mu \mathrm{m}$ emission comprises line emission from PAH molecules and a continuum component from warm dust particles heated by Ly- $\alpha$ and UV radiation, the prime source of which are young star clusters that will have moved further downstream of the spiral arm shock. Figures 5 and 6 clearly show, for the first time that we are aware of, the systematic offset of dense gas spiral arms and infrared arms along regions several kpc long.

Second, the I6 spiral arms are generally offset on the concave side of the ISO arms, by $200-300$ pc. There is also a tendency for $\mathrm{I} 6$ to sit upstream of the $\mathrm{CO}$ arms inside $\sim 6 \mathrm{kpc}$ radius, by around $100-200 \mathrm{pc}$. This offset is harder to explain. Thermal radio emission will be enhanced by the same new star clusters 

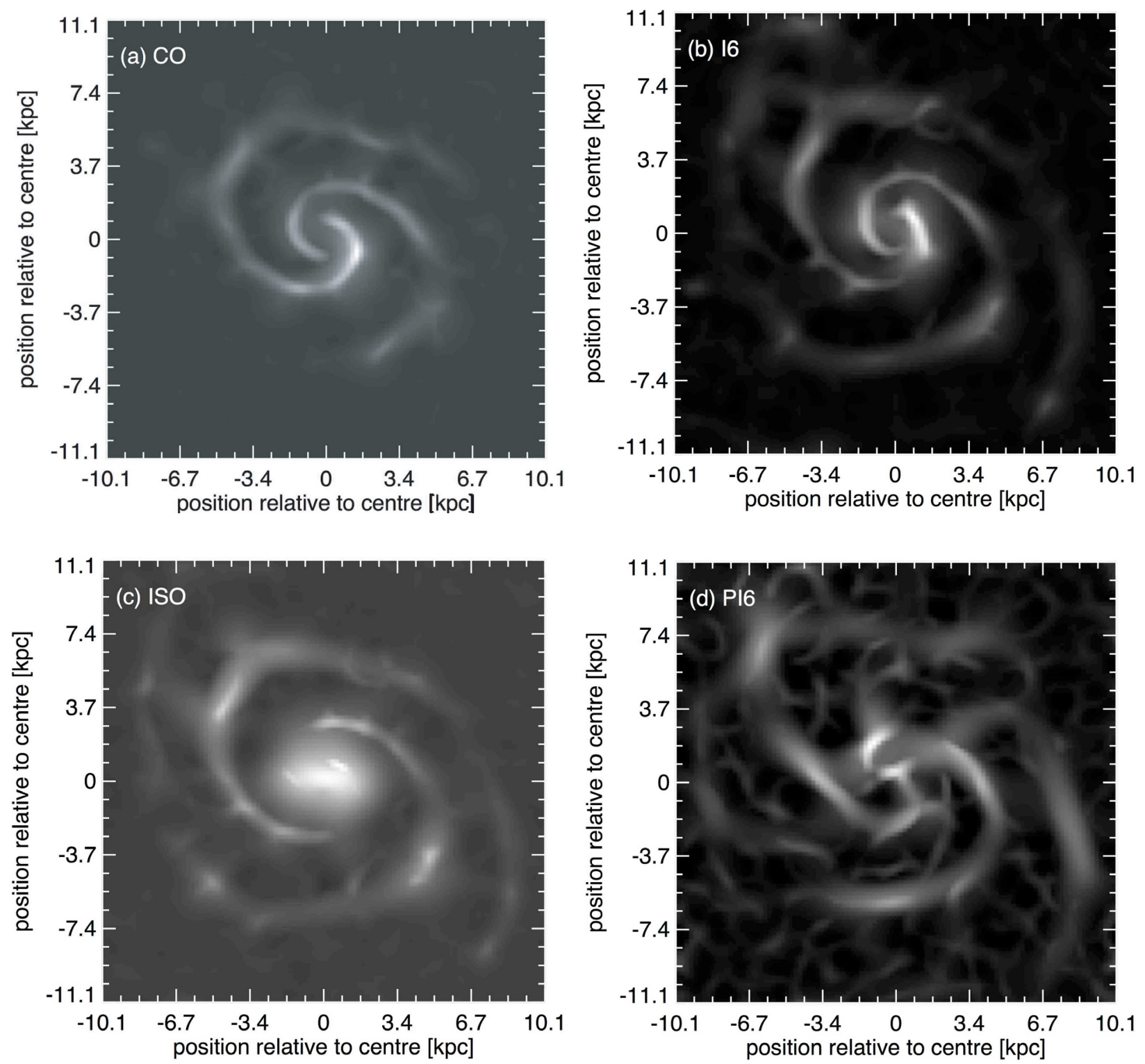

Fig. 4. The maximum anisotropic-wavelet coefficients of the original maps shown in Fig. 3. a) $\mathrm{CO}(1-0)$ emission; b) total radio emission at $\lambda 6 \mathrm{~cm}$; c) infrared ISO $15 \mu \mathrm{m}$ emission; d) polarized $\lambda 6 \mathrm{~cm}$ radio emission. The grey-scale is proportional to the amplitude of the wavelet coefficient (white is high). At each location the wavelet coefficient was calculated for a fixed range of scales and 180 orientations, the highest coefficient is shown so the wavelet scale may be different between pixels.

that heat the dust and PAHs. Hence no offset between the I6 and ISO arms is expected from thermal effects. The most significant non-thermal process (on $\sim 100 \mathrm{pc}$ scales) associated with star formation is probably an increase in total magnetic field strength, caused by turbulent tangling of magnetic field lines. At our $8^{\prime \prime}$ resolution $(\sim 400 \mathrm{pc})$ this synchrotron emission will be predominantly unpolarized, again tending to align the I6 and ISO ridges. The shift of the I6 arms upstream of the ISO arms can be due to the polarized synchrotron emission.

The polarized ridge in the inner part of arm 2 is clearly located in the inter-arm region and may have no direct connection with the gaseous arms (see below), but along most of the inner part of arm $1, r \lesssim 6 \mathrm{kpc}$, the polarized ridge is close to the CO ridge, sometimes very closely aligned but often shifted about 300 pc upstream. This displacement of the PI6 arm 1 indicates that the regular magnetic field is strongest upstream of the $\mathrm{CO}$ arm, and the PI6 ridge is shifted further upstream than the I6 ridge. At radii of 6-8 kpc the PI6 ridges of arm 1 and arm 2 move from the concave to the convex side of the other tracers. The shift was also seen in the $\lambda \lambda 18,20 \mathrm{~cm}$ polarization maps of Horellou et al. (1992). Beyond this radius, the two polarization arms behave differently: along the outer part of arm 2 the polarization ridge is intertwined with the I6 and ISO, whereas along the outer part of arm 1 the PI6 sits consistently on the convex side of the I6 and ISO ridges. $\mathrm{CO}$ is absent at these large radii. The differences between the inner and outer spirals are difficult to explain, but may be related to the conjunction of an inner spiral density wave and an outer material spiral pattern identified by Elmegreen et al. (1989), overlapping in the range $6 \mathrm{kpc} \lesssim r \lesssim 8 \mathrm{kpc}$. The largest shifts between PI6 and other tracers occur on the side of M 51 closest to the companion galaxy, which may have caused the two outer spirals (Howard \& Byrd 1990).

The offset of the PI6 ridge upstream of the $\mathrm{CO}$ ridge in arm 1 in the inner disc indicates that the spiral shock lies slightly upstream of the $\mathrm{CO}$ ridges. The position of the $\mathrm{I} 6$ ridge is then 

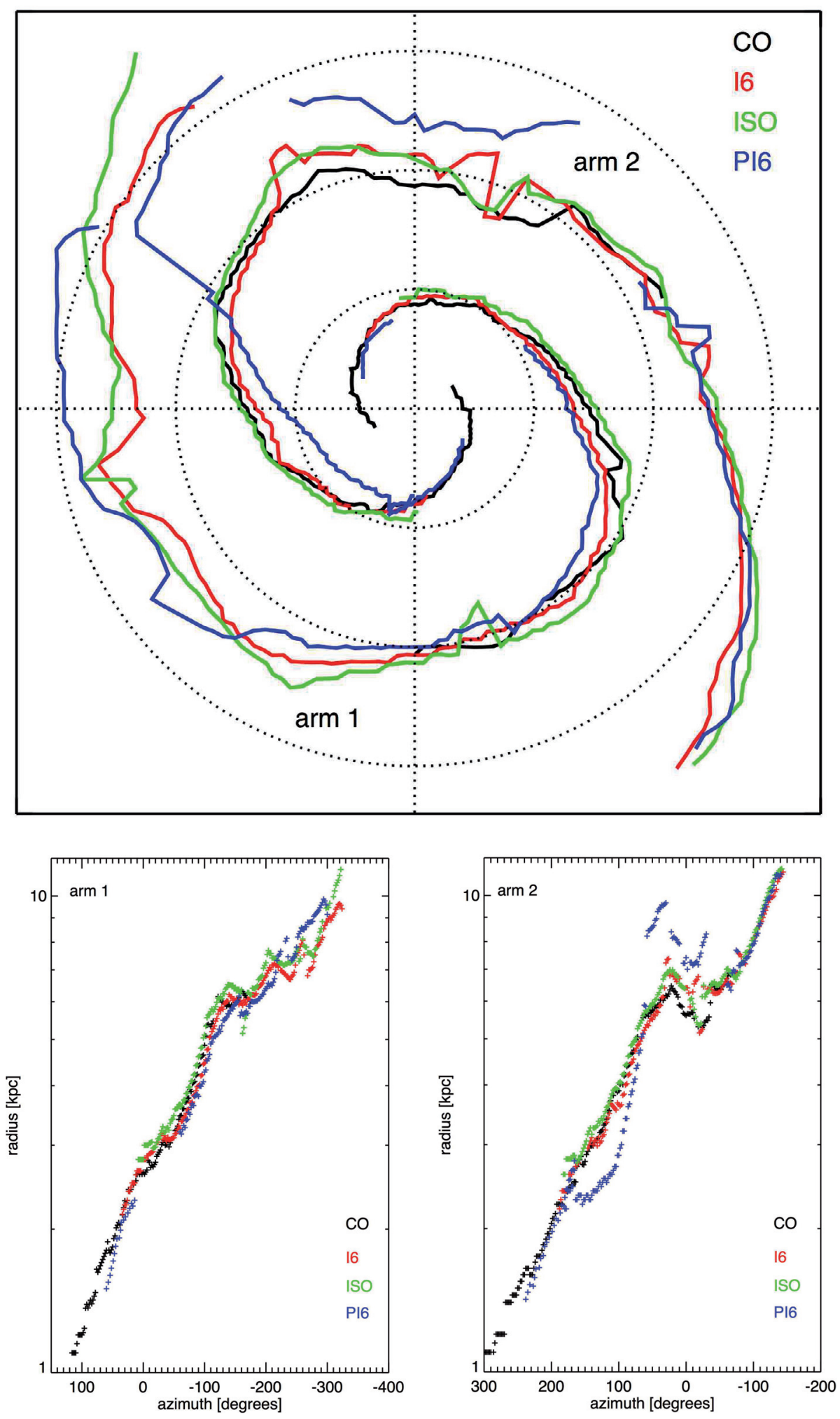

Fig. 5. Location of the various spiral arm ridges in the plane of M 51; dotted lines show the galacto-centric radii 3, 6 and $9 \mathrm{kpc}$. I6 and PI6 are the total and polarized radio intensities at $\lambda 6 \mathrm{~cm}$, ISO the $15 \mu \mathrm{m}$ infrared emission. The ridges are the positions where the anisotropic wavelet coefficient has a maximum, tracking along the spiral arms, as described in the text.

Fig. 6. Radius (on a logarithmic scale) versus azimuth plots of the spiral arm ridges. This shows the regions of systematic offsets in ridges quite well (compare to Fig. 5). The azimuthal angle is measured counter-clockwise from the top of the face-on galaxy. due to a balance between the polarized (non-thermal) emission pulling it upstream of the $\mathrm{CO}$ and the thermal and unpolarized emission pulling it towards the ISO ridge.

Third, radio polarization arms are partly situated in interarm regions, most noticeably over $\sim 3 \mathrm{kpc}$ in the inner part of arm 2 and north of the middle part of arm 2 and in the outer part of arm 1, where they are displaced by around $700 \mathrm{pc}$ from the other arms. Polarized intensity maps at $\lambda \lambda 3,6 \mathrm{~cm}$ (Fletcher et al. in preparation) clearly show concentrations of emission in these inter-arm regions, particularly in the inner part of arm 2: we emphasise that these elongated polarized structures are not artefacts caused by the wavelet analysis. These sections of the PI6 ridges resemble the magnetic arms observed in NGC 6946 (Beck \& Hoernes 1996). However, the PI6 ridge that is associated with arm 2 near the galaxy's centre forms a continuous structure that is initially well aligned with the $\mathrm{CO}$ ridge then, as the radius increases, moves into the inter-arm region, rejoins arm 2 and then crosses inter-arm space again to link up with 
arm 1. This interlacing of large scale magnetic spiral structures and gaseous arms is quite unlike the behaviour of the magnetic arms seen in NGC 6946, but resembles that in NGC 2997 (Han et al. 1999). Higher resolution and sensitivity may reveal similar structures in other galaxies. Its explanation will require more sophisticated modelling of the interplay between spiral density waves and magnetic fields than has been used to date and is beyond the scope of this paper.

\subsection{Variation of pitch angles along the spiral arms}

Figures 7 and 8 show how the pitch angles of the spiral arms vary along their length. The uncertainties in pitch angles tend to get greater with increasing distance along the arms; the generally fainter emission (compared to the inner disc) means that the maximum wavelet coefficients are smaller and the arms become less well defined (Fig. 4). First we will discuss the pitch angles for each spiral arm tracer in turn, then we will compare the pattern of pitch angles in different tracers.

The two CO spiral arms can be traced through $\Delta \phi \approx 360^{\circ}$ in azimuth. In both $\mathrm{CO}$ arms the pitch angle in the inner quarter turn $\left(\Delta \phi \approx 90^{\circ}\right)$ is approximately constant, within the errors, but the pitch angles of the two arms in this region differ by $\sim 10^{\circ}$ : in $\operatorname{arm} 1, p_{\mathrm{CO}} \approx 25^{\circ}$ and in arm $2, p_{\mathrm{CO}} \approx 15^{\circ}$. Further along the arms the pitch angles follow different trends. Thus, despite the apparent similarity of the two $\mathrm{CO}$ arms, they are not symmetric under rotation. There is substantial variation of $p_{\mathrm{CO}}$ in both arms, with ranges $0^{\circ} \lesssim p_{\mathrm{CO}} \lesssim 30^{\circ}$ in arm 1 and $-20^{\circ} \lesssim p_{\mathrm{CO}} \lesssim 40^{\circ}$ in arm 2. There is a tendency for low or negative pitch angles to occur toward the ends of both arms. This shift in pitch angles to low and even negative values occurs at $\phi \approx-100^{\circ}$ in arm 1 and $\phi \approx+60^{\circ}$ in arm 2 . The transition occurs around the place where the character of the CO arms - especially arm 2 - markedly changes in the original map (Fig. 3). The galacto-centric radii of these positions are around $6 \mathrm{kpc}$, which is the approximate position of the co-rotation radius (Elmegreen et al. 1992). At these azimuths the pitch angles of the other tracers show similar variations. Furthermore in the azimuthal ranges $40^{\circ}>\phi>-100$ in arm 1 and $200^{\circ}>\phi>60^{\circ}$ in arm 2 the maxima and minima in $p_{\mathrm{CO}}$ follow each other with the same interval in $\phi$; changes in $p_{\text {CO }}$ occur symmetrically along the two arms, but with different magnitudes.

The $\lambda 6 \mathrm{~cm}$ total radio emission (I6) and ISO $15 \mu \mathrm{m}$ spirals are both quite well defined over the first half turn $\left(\Delta \phi \approx 200^{\circ}\right)$ with systematic trends in their pitch angles. The variation in pitch angles is stronger than in the $\mathrm{CO}$ arms and the uncertainty in the outer arm pitch angles is higher; this is because the arms are better defined in CO, whereas in I6 and ISO the arms are broader and contain bright patches, probably arising from regions of intense star formation. The $\lambda 6 \mathrm{~cm}$ polarization arms (PI6) are generally the least well defined of all, although the first $\Delta \phi \approx 130^{\circ}$ of arm 2 is very clear and shows a sharp and systematic variation in pitch angle from $p_{\mathrm{PI}} \simeq 30^{\circ}$ to $p_{\mathrm{PI}} \simeq 0^{\circ}$ and back up to $p_{\text {PI }} \simeq 60^{\circ}$. Both PI6 arms show strong variations in pitch angle - a range of $-20^{\circ} \lesssim p_{\text {PI }} \lesssim 60^{\circ}$ in arm 2 .

In Sect. 4.2 we saw that the position of the spiral arm ridges is not the same in all tracers, but that there can be small but systematic shifts in the arm location. Now we consider how the pitch angles compare along arms.

In general there is quite good agreement and $p_{\mathrm{CO}} \approx p_{\mathrm{I}}$, particularly in arm 1 for $\phi>-120^{\circ}$ where the pitch angles agree within errors for $\Delta \phi \approx 100^{\circ}$. In arm 2 there are alternating regions of good and bad agreement in pitch angles. Overall, the similarity in pitch angles and locations where both tracers show
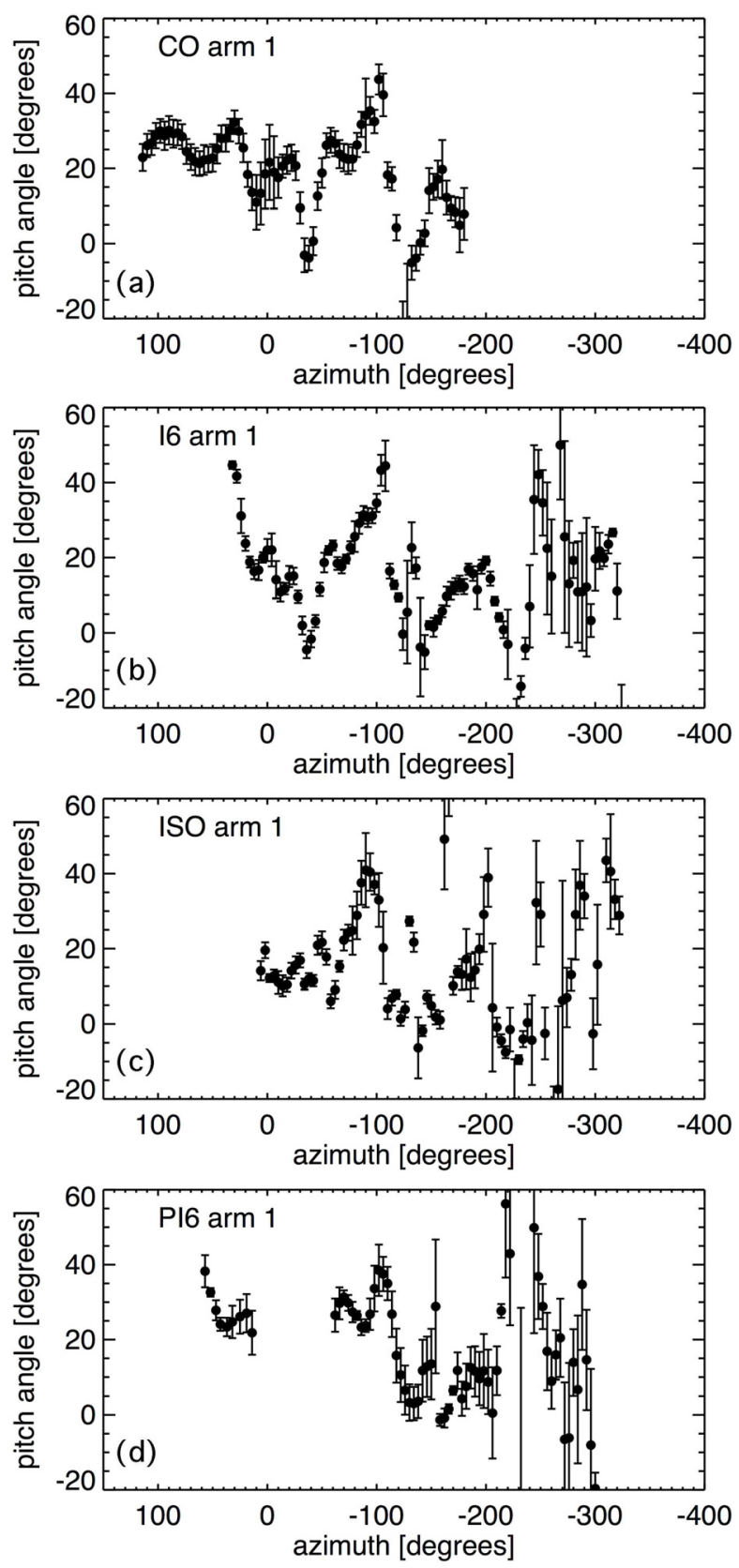

Fig. 7. Pitch angles of arm 1 (see Fig. 3 for arm labelling). a) $\mathrm{CO}(1-0)$ emission. b) I6 $\lambda 6 \mathrm{~cm}$ total emission. c) ISO $15 \mu \mathrm{m}$ emission. d) PI6 $\lambda 6 \mathrm{~cm}$ polarized emission. Every $6^{\circ}$ in azimuth (measured counter-clockwise from the top of the face-on galaxy) along an arm, the pitch angle of the wavelet with the highest amplitude is shown. Errors are calculated according to the Monte Carlo method described in Sect. 4.1. The arms run from left (smallest radius) to right (largest radius) in all panels.

changes to higher or lower pitches, the physical process producing the $\mathrm{CO}$ arms is likely the same as that producing the I6 arms, as expected for density wave compression.

Finally we compare $p_{\text {I }}$ and $p_{\text {ISO }}$. In arm 2 the angles are for the most part equal, and where they are not the tendency (i.e. whether there is a decrease or an increase in pitch angle) is the same. Thus, despite the systematic small offset in the location of the I6 and ISO arms, their pitch angles are apparently modified for the same reasons. 

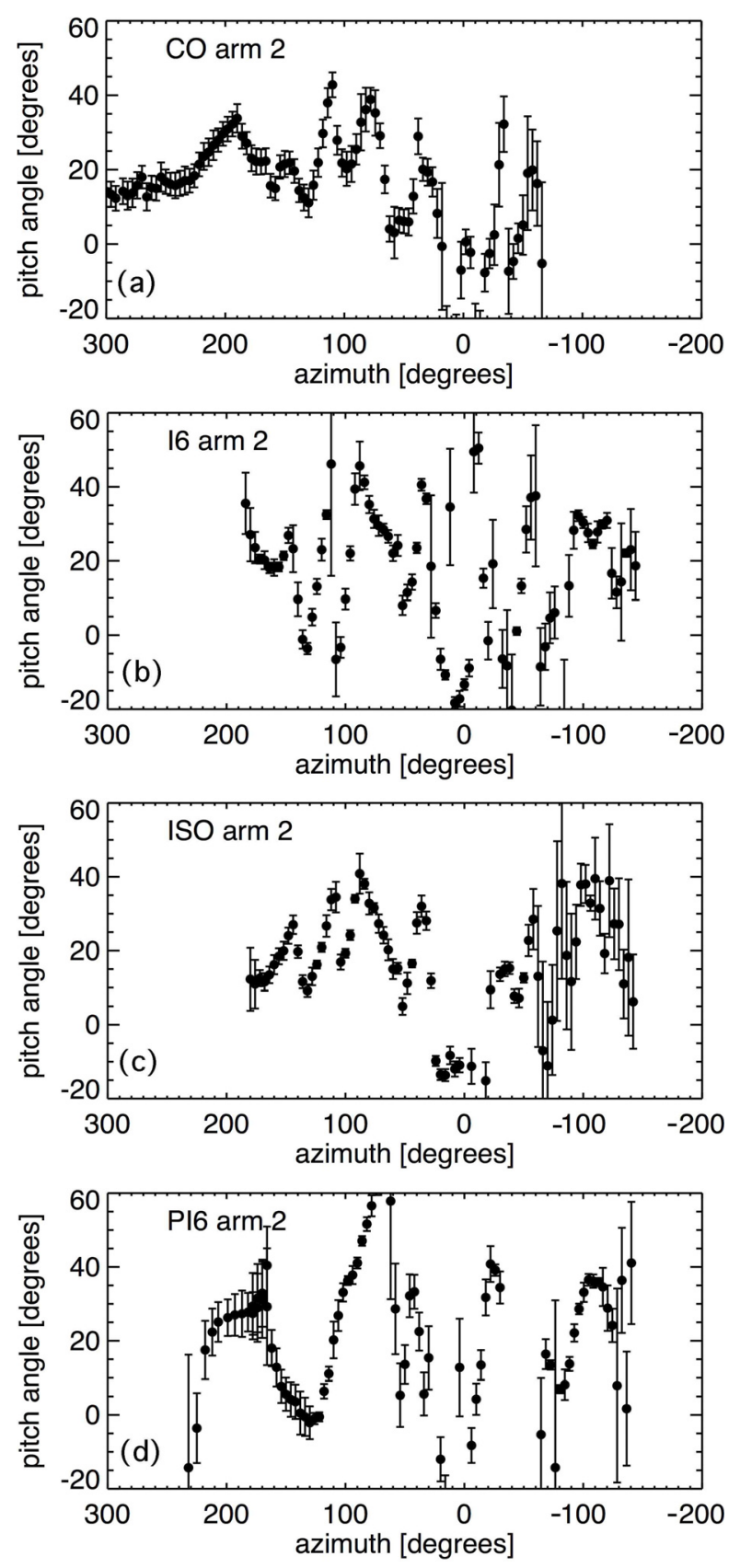

Fig. 8. As in Fig. 7 but for arm 2.

\subsection{Orientation of the regular magnetic field and pitch angles of the gaseous and magnetic arms}

In this section we will compare the orientation of the regular magnetic field lines with the pitch angles of the gaseous spiral structure (traced by $\mathrm{CO}$ emission) and with the pitch angles of the spiral structure in polarization (PI6).

The intrinsic orientation of the regular magnetic field $\left(p_{B}\right)$ was determined from the observed Stokes $Q$ and $U$ values by using observations at $\lambda 3.5 \mathrm{~cm}$ and $\lambda 6.2 \mathrm{~cm}$ to correct for Faraday rotation. The errors in magnetic field pitch angles were calculated using the noise in the observed maps only and do not include any systematic effects, such as uncertainty in the galaxy inclination (such systematic effects will equally effect the wavelet derived spiral arm pitch angles and so should not invalidate our comparisons). We only plot $p_{B}$ where the polarized intensity at both wavelengths is greater than three times the noise level. In order to increase the signal to noise ratio and thus to see the azimuthal trends in $p_{B}$ better, we have used $Q$ and $U$ data smoothed to $15^{\prime \prime}$ (the anisotropic wavelet effectively smoothes the original $8^{\prime \prime}$ data in seeking the optimum position and orientation of the spiral arms). However, we have also calculated $p_{B}$ at $8^{\prime \prime}$ resolution and the main results - concerning the alignment or misalignment of $p_{B}$ with $p_{\mathrm{CO}}$ and $p_{\mathrm{PI}}-$ do not change.

Figure 9 shows how the orientation of the magnetic field lines varies with azimuthal angle at radii in the range $0.9 \mathrm{kpc}<$ $r<6.5 \mathrm{kpc}$. The pitch angles of the two CO spiral arms are also indicated (see Sect. 4.1). These show the positions of the gaseous spiral arms and let us measure how well the orientations of the magnetic field lines and spiral structure agree.

The first thing to note is that the orientation of the magnetic field lines is almost never zero. This means that the regular magnetic field has a spiral shape - and therefore both a radial and an azimuthal component - virtually everywhere in the region shown. The orientation of the regular magnetic field is consistently less than $45^{\circ}$ at the radii shown $\left(p_{B}>45^{\circ}\right.$ only for small regions at $r>5.3 \mathrm{kpc}$ ); the azimuthal component of the magnetic field dominates the radial component for $r<6.5 \mathrm{kpc}$.

Figure 9 also shows that the orientation of the regular magnetic field lines vary by at least $\sim 30^{\circ}$ in each ring; this is by no means obvious in maps showing the $\boldsymbol{B}$-vectors (e.g. Fig. $3 \mathrm{~d}$ ), where the field orientation appears rather constant. The same situation may also exist in observations of other external galaxies, whereby the field appears to be better aligned with the optical spiral and have a more consistent orientation than is really the case. Note that even for galaxies with a small inclination to the line of sight a slice at constant radius is quite elliptical and it is hard to judge "by sight" how the orientation of the field varies with azimuth.

The other striking feature of Fig. 9 is the extremely good agreement between the $\mathrm{CO}$ spiral arm pitch angles and the magnetic field orientation at the position of the $\mathrm{CO}$ arms. This cannot be a coincidence as $p_{B}$ itself varies considerably at each radius and the agreement holds whether $p_{\mathrm{CO}}$ is low (e.g. $p_{\mathrm{CO}} \approx 20^{\circ}$ at $r=1.6 \mathrm{kpc}$ ) or high (e.g. $p_{\mathrm{CO}} \approx 40^{\circ}$ at $r=5.0 \mathrm{kpc}$ ). Thus the orientation of the regular magnetic field and the gaseous spiral arms are tightly linked in M 51. There is not a consistent trend in $p_{B}$ away from the spiral arms. The magnetic field orientation is sometimes higher and sometimes lower in the inter-arm region than at the position of the gaseous spiral arms.

Berkhuijsen et al. (1997) found a broad agreement between optical arm pitch angles (Howard \& Byrd 1990), averaged in $\sim 5 \mathrm{kpc}^{2}$ sectors, and their large scale magnetic field model of M 51 .

In Fig. 10 we compare $p_{B}$ with $p_{\mathrm{PI}}$. In polarization arm 1 $p_{B} \simeq p_{\mathrm{PI}}$; the regular magnetic field orientation is parallel to the ridge of the PI6 arm. This indicates that the same physical effect is responsible for both the increase in polarized emission along the ridge and the orientation of the regular magnetic field there. This section of polarization arm 1 lies $100-200$ pc upstream of the corresponding arms in other tracers. In contrast, along polarization arm $2, p_{B}<p_{\mathrm{PI}}$; the magnetic field always has a lower pitch angle than the polarization arm, with a difference in angle of $10-20^{\circ}$. This section of polarization arm 2 lies about $\sim 400 \mathrm{pc}$ upstream of the other tracers of arm 2 and proceeds to cross over the gas and optical spirals at a radius of $r \simeq 5.5 \mathrm{kpc}$. This indicates that different forces are at work in producing polarization arms 1 and 2. 

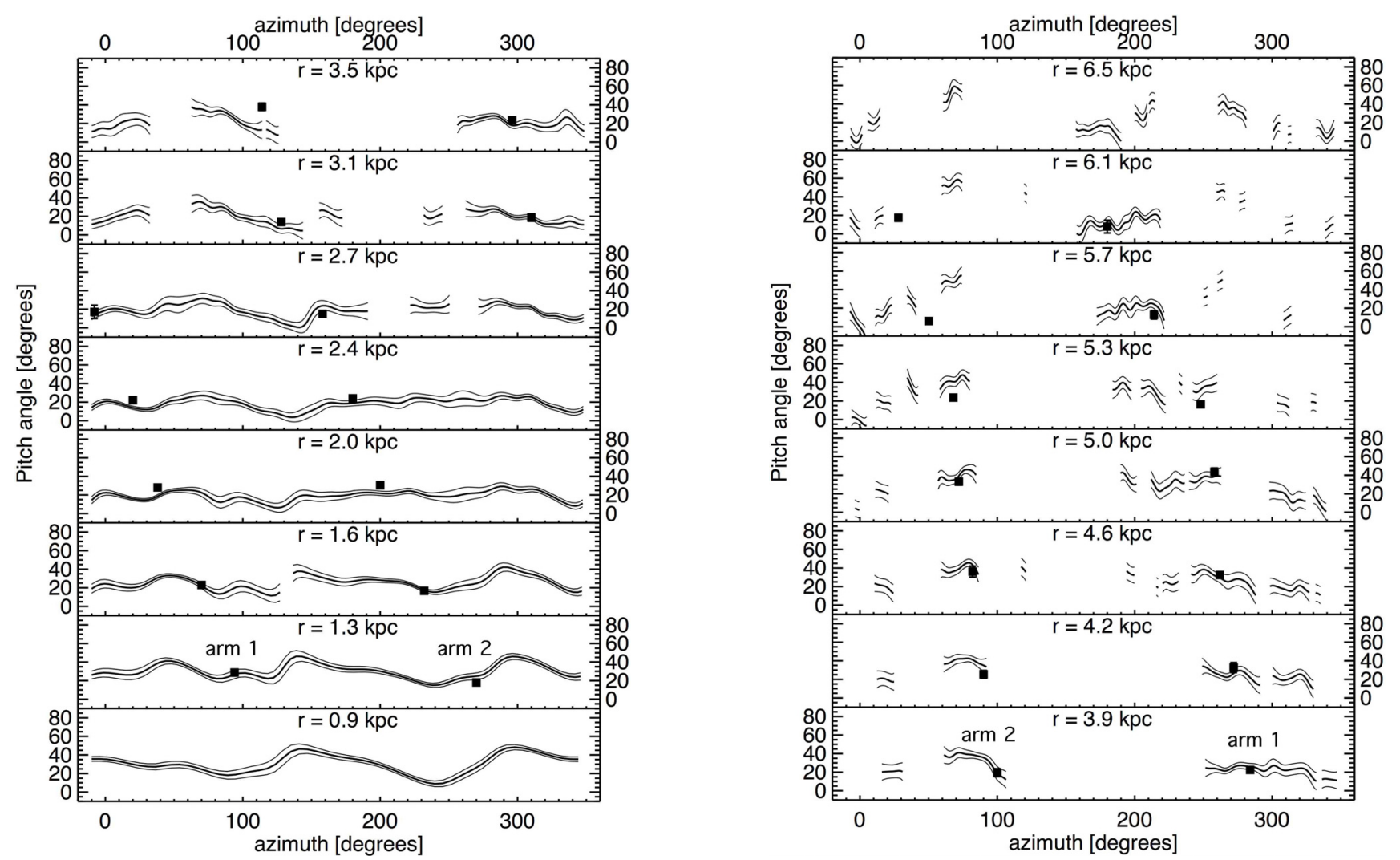

Fig. 9. Azimuthal variation of the orientation of the regular magnetic field $p_{B}=1 / 2 \arctan (Q / U)$, corrected for Faraday rotation, plotted as curves at different radii from the centre of M 51. Uncertainties are shown by upper and lower curves. The resolution of the observations is $15^{\prime \prime}$. Angles are only plotted where the polarized intensity at both $\lambda 3 \mathrm{~cm}$ and $\lambda 6 \mathrm{~cm}$ is above $3 \times$ the noise. Also shown are pitch angles of the CO arms (filled squares with error bars - most errors are slightly smaller than the size of the squares). The azimuthal angle is measured counter-clockwise from the top of the face-on galaxy and the gas flow relative to the spiral pattern is from left to right.

\section{Discussion}

New observations of the spiral galaxy M 51, obtained with the VLA and Effelsberg radio telescopes, and published maps of the molecular $(\mathrm{CO})$ and mid-infrared $(15 \mu \mathrm{m})$ emissions, were used to compare the spiral patterns present in the magnetic field, dense gas and dust distributions.

1. We have shown that anisotropic wavelet functions are appropriate tools to locate the positions of spiral arms in images of galaxies and to measure their local pitch angles.

2. A systematic offset between the spiral ridges of molecular gas (CO) emission and of mid-infrared (ISO $15 \mu \mathrm{m}$ ) emission can be followed over many kpc along the arms of M 51 (Figs. 5 and 6). The typical offset is $100 \mathrm{pc}$ corresponding to a typical time delay of $\sim 10^{7} \mathrm{yr}$ for gas well inside the co-rotation radius, assuming a circular rotation velocity of $200 \mathrm{~km} \mathrm{~s}^{-1}$ (Garcia-Burillo et al. 1993), pattern speed of $38 \mathrm{~km} \mathrm{~s}^{-1} \mathrm{kpc}^{-1}$ (Zimmer et al. 2004) and arm inclination of $20^{\circ}$. The delay can be interpreted as the interval between the formation of Giant Molecular Clouds (GMCs) and the appearance of newly formed stars. A delay of $\sim 10^{7} \mathrm{yr}$ between dense gas accumulation and star formation was also estimated by Tosaki et al. (2002) from an analysis of the location of ${ }^{12} \mathrm{CO}$ and ${ }^{13} \mathrm{CO}$ emission lines.

We do not see a shift in the relative positions of the ISO and $\mathrm{CO}$ arms, i.e. the $\mathrm{CO}$ arm does not move from the concave to the convex side of the ISO arm, near to the co-rotation radius of $r \simeq 6.2 \mathrm{kpc}$ determined by Elmegreen et al. (1992). This may be due to the non-trivial dynamical effects of two, overlapping, spiral arm patterns in M 51 (Elmegreen et al. 1989).
3. We also found a systematic offset between the spiral ridges of total radio continuum emission and those of mid-infrared and $\mathrm{CO}$ emission, indicating a shift between regions of strong thermal and non-thermal radio continuum emission. While the thermal component is expected to closely follow the distribution of star-forming regions as traced by mid-infrared emission, the non-thermal component is probably enhanced due to compression of magnetic fields in a spiral shock front, located upstream of the mid-infrared spiral arm. Cosmic ray electron density is expected to be higher on the downstream side of the arms, where supernova remnants should be more common, but on scales of a few hundred parsecs this may not have a significant effect on the synchrotron intensity; we are assuming that the distribution of cosmic ray electrons is mainly determined by the magnetic field, rather than the distribution of their sources. The purely non-thermal, polarized radio ridges lie the furthest upstream of all the data as expected in this scenario. This is in agreement with the results of Tilanus et al. (1988) who found a general arm sequence of: non-thermal emission - (cold) dust lane - thermal emission $-\mathrm{H} \alpha$.

Inside co-rotation our observations reveal the following sequence from upstream to downstream: polarized radio emission, total radio emission, $\mathrm{CO}$, infrared emission, interpreted as a result of the sequence of components: shock-compressed magnetic field, molecular gas clouds, and UV heated (warm) dust. This can arise if the spiral shock is located slightly ahead of the $\mathrm{CO}$ ridge, a plausible consideration if molecule formation and/or cloud condensation are triggered by the spiral shock. The compression of magnetic field, resulting in stronger polarized 


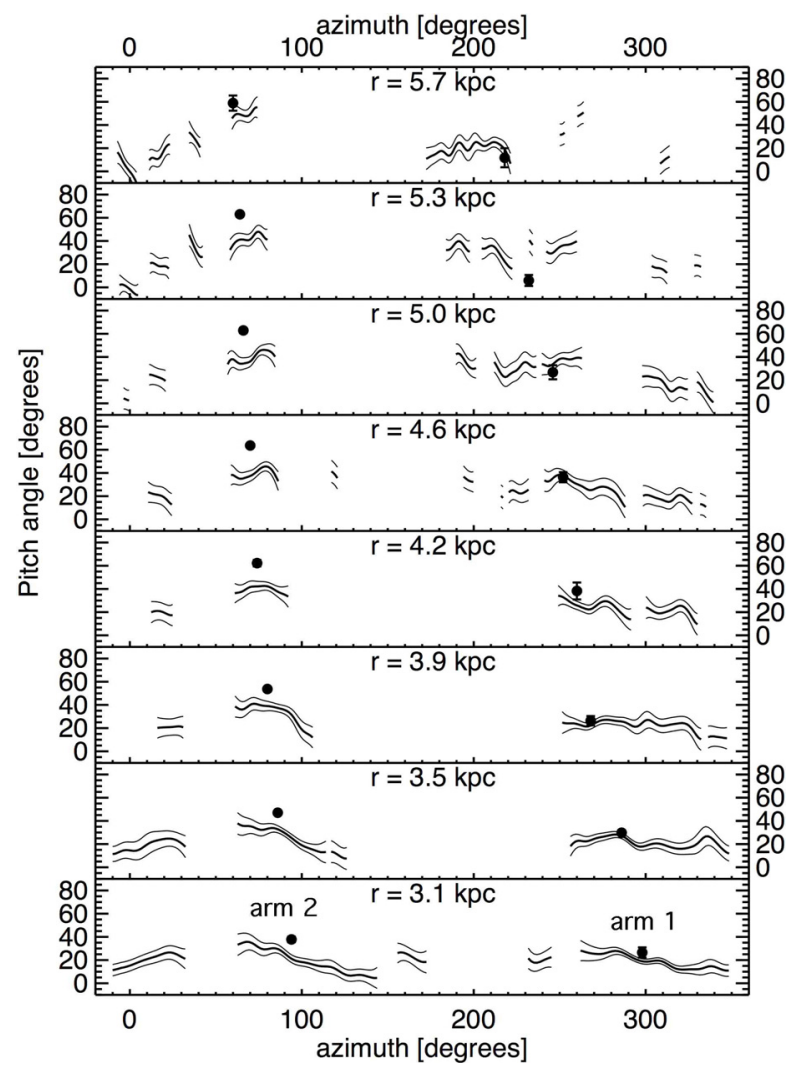

Fig. 10. As in Fig. 9, lines show the orientation of the regular magnetic field, but the filled circles with error bars represent the pitch angle of the ridges of $\lambda 6 \mathrm{~cm}$ polarized emission, $p_{\mathrm{PI}}$. The polarized ridge running from azimuth $\sim 100^{\circ}$ at the bottom to $\sim 60^{\circ}$ at the top is the prominent inter-arm polarization ridge in Fig. 5.

radio emission, occurs at the shock, but the formation of dense $\mathrm{CO}$ clouds is only completed a few hundred parsec downstream. Following similar arguments to those used in point 1 above, we can estimate the time delay between gas entering the shock front and the accumulation of dense GMCs: a typical polarized ridge offset of $300 \mathrm{pc}$ corresponds to an interval of $\sim 10^{7} \mathrm{yr}$. The total time between gas entering the spiral shock and the emergence of newly formed stars will then be a few tens of millions of years.

The above discussion only applies to a diffuse component of the $\mathrm{CO}$ in the inter-arm region, which can be compressed by a large scale shock at the spiral arm. Molecular gas that is already gravitationally bound in clouds will not respond to the shock. Extended, faint $\mathrm{CO}(1-0)$ emission in the inner inter-arm region of M 51 is clearly seen in the combined single-dish and interferometer data of Helfer et al. (2003), suggesting that at least a part of the molecular gas is diffuse. However the higher resolution, interferometer only, CO(1-0) maps of Aalto et al. (1999) reveal small clumps of inter-arm molecular gas, so that some - difficult to estimate - fraction of the upstream gas is probably already confined in clouds. This means that the shock strength will be difficult to infer from gas density ratios in the inter-arm and arm regions. On the other hand, Aalto et al. could identify steep velocity gradients both in and, particularly clearly, between giant associations of molecular gas which are in broad agreement with theoretical expectations of spiral density wave shocks. The detailed physics of the gas response to possible shocks in M 51 and the effect this has on the spiral arm morphology requires more sophisticated modelling than we can attempt here, especially the apparent upstream shift of the shock in the magnetic field; there are, however, strong indications that large scale spiral shocks occur in the vicinity of the gas arms and that these compress diffuse gas.

4. The maintenance of both radial and azimuthal components of the magnetic field, and hence a non-zero pitch angle, is an important prediction of dynamo theory (Beck et al. 1996; Shukurov 2000) but is very difficult to explain if the magnetic field is purely passive and stretched by shear in the galactic differential rotation, since after a small number of galactic rotations the field would be completely circular, i.e. it would have a pitch angle $p_{B} \simeq 0$.

5. Although the $\boldsymbol{B}$-vectors of polarized radio emission from M 51 seem to have the same orientations as the CO arms (Fig. 3), they smoothly change by about $\pm 15^{\circ}$ around any fixed radius, without a systematic trend between the arm and inter-arm regions (Fig. 9). Smaller pitch angles in inter-arm regions are predicted by some dynamo models and have been observed e.g. in NGC 6946 (Rohde et al. 1999). In M 51, however, the regular magnetic field structure is probably dominated by streaming motions and compression in the spiral shock (see below). The field is brought into good alignment at the $\mathrm{CO}$ arms or vice versa (Fig. 9) and then relaxes into a different configuration in the inter-arm regions that depends on e.g. the amount of compression in the arms, the energy of turbulent flows, the orientation of dynamo-generated field modes etc.

6. We found a close alignment of the $\mathrm{CO}$ pitch angles and the regular magnetic field orientation at the position of the arms (Fig. 9). This could be due to shock amplification of the regular field component parallel to the shock.

If the field is frozen into the gas then $B_{\|}^{(s)}=\epsilon B_{\|}$and $B_{\perp}^{(s)}=$ $B_{\perp}$, where $\epsilon=\rho^{(s)} / \rho$ is the compression ratio in the gas density $\rho$ and the superscript $(s)$ labels quantities at the shock front. The deflection of the magnetic field $\Delta \theta$, where $\theta$ is the angle between the field and the shock front upstream of the shock, is given by

$$
\begin{aligned}
\Delta \theta & =\theta-\theta^{(s)}=\arctan \left(\frac{B_{\perp}}{B_{\|}}\right)-\arctan \left(\frac{B_{\perp}}{\epsilon B_{\|}}\right) \\
& \simeq \frac{B_{\perp}}{B_{\|}}\left(1-\frac{1}{\epsilon}\right)
\end{aligned}
$$

where the last line holds for $B_{\perp} / B_{\|} \ll 1$. For $\epsilon=4$ (maximum degree of gas compression by an adiabatic shock) and $B_{\perp} / B_{\|}=$ 0.5 (i.e. the magnetic field is initially inclined at $30^{\circ}$ to the shock front, a conservative estimate cf. Fig. 9) we obtain $\Delta \theta \simeq 20^{\circ}$. Thus a strong spiral shock can be expected to align the regular magnetic field rather well with the spiral arm.

If the shock is weak $(\epsilon<4)$ or the upstream field makes a larger angle with the shock front then an additional source of alignment is required. The conversion of an isotropic random magnetic field component upstream of the shock into an anisotropic random field in the shocked region (where the anisotropic random field will be perfectly aligned with the shock) provides such a mechanism. Strong polarized emission arising from anisotropic random magnetic fields formed in shearing shocks has recently been identified in the barred galaxies NGC 1097 and NGC 1365 (Beck et al. 2005).

7. Some of the polarized radio emission (and hence some of the regular magnetic fields) forms ridges which are interlaced with the gaseous arms: the polarized ridge of arm 2 clearly lies in between gas arms 1 and 2 south-east of the central region. The magnetic field is not oriented parallel to this polarization arm but is inclined to the axis of the arm by up to $20^{\circ}$ (Fig. 10). In contrast to polarization arm 1 , spiral shock compression does 
not seem to be the origin of these enhanced regular magnetic fields. The field orientation seems to be between that of the polarization arm and that of the nearby $\mathrm{CO}$ arm 2, like finding a compromise between two different forces. One force causes the polarization arm to cross $\mathrm{CO}$ arm 2 to join the outer arm 1, the second tries to align the field with the gas arm.

Acknowledgements. We thank Anvar Shukurov for useful discussions and the referee for helpful comments. This work was supported in part by the DFGRFBR (grant 03-02-04031). I.P. is grateful to the Max-Planck-Institut für Radioastronomie for support and hospitality. A.F. acknowledges the Leverhulme Trust for financial support under research grant $\mathrm{F} / 00125 / \mathrm{N}$. This research made use of NASA's Extragalactic Database (NED) and Astrophysical Data System (ADS).

\section{References}

Aalto, A., Hüttermeister, S., Scoville, N. Z., \& Thaddeus, P. 1999, ApJ, 522, 165 Antoine, J.-P., Carette, P., Murenzi, R., \& Piette, B. 1993, Signal Processing, 31, 241

Beck, R. 2005, in Cosmic Magnetic Fields, ed. R. Wielebinski, \& R. Beck (Berlin: Springer), 41

Beck, R., \& Hoernes, P. 1996, Nature, 379, 47

Beck, R., Brandenburg, A., Moss, D., Shukurov, A., \& Sokoloff, D. 1996, ARA\&A, 34, 155

Beck, R., Fletcher, A., Shukurov, A., et al. 2005, A\&A, 444, 739

Berkhuijsen, E. M., Horellou, C., Krause, M., et al. 1997, A\&A, 318, 700

Elmegreen, B. G., Elmegreen, D. M., \& Seiden, P. E. 1989, ApJ, 343, 602

Elmegreen, B. G., Elmegreen, D. M., \& Montenegro, L. 1992, Ap\&SS, 79, 37

Frick, P., Beck, R., Shukurov, A., et al. 2000, MNRAS, 318, 925

Frick, P., Beck, R., Berkhuijsen, E. M., \& Patrikeev, I. 2001, MNRAS, 327, 1145

García-Burillo, S., Guélin, M., \& Cernicharo, J. 1993, A\&A, 274, 123
Han, J. L., Beck, R., Ehle, M., Haynes, R. F., \& Wielebinski, R. 1999, A\&A, 348,405

Helfer, T. T., Thornley, M. D., Regan, M. W., et al. 2003, ApJS, 145, 259

Henry, A., Quilien, A. C., \& Gutermuth, R. 2003, AJ, 126, 2831

Holschneider, M. 1995, Wavelets: An Analysis Tool (Oxford: Oxford University Press)

Horellou, C., Beck, R., Berkhuijsen, E. M., Krause, M., \& Klein, U. 1992, A\&A, 265,417

Howard, S., \& Byrd, G. 1990, AJ, 99, 1990, 1798

Kennicutt, R. C. 1981, AJ, 86, 1847

Knapen, J. H., Beckman, J. E., Cepa, J., van der Hulst, T., \& Rand, R. J. 1992, ApJ, 385, L37

Meijerink, R., Tilanus, R. P. J., Dullemond, C. P., Israel, F. P., \& van der Werf, P. P. 2005, A\&A, 430, 427

Patrikeev, I., Fletcher, A., Beck, R., et al. 2005, in The Magnetized Plasma in Galaxy Evolution, ed. K. T. Chyży, K. Otmianowska-Mazur, M. Soida, \& R.-J. Dettmar (Kraków), 130

Petit, H., Hua, C. T., Bersier, D., \& Courtès, G. 1996, A\&A, 309, 446

Rand, R. J., \& Kulkarni, S. R. 1990, ApJ, 349, L43

Roberts, W. W. 1969, ApJ, 158, 123

Rohde, R., Beck, R., \& Elstner, D. 1999, A\&A, 350, 423

Sandage, A., \& Tammann, G. A. 1974, ApJ, 194, 559

Sauvage, M., Blommaert, J., Boulanger, F., et al. 1996, A\&A, 315, L89

Shu, F. H., Milione, V., Gebel, W., et al. 1972, ApJ, 173, 557

Scoville, N. Z., Polletta, M., Ewald, S., et al. 2001, AJ, 122, 3017

Shukurov, A. 2000, in The Interstellar Medium in M31 and M33, ed. E. M. Berkhuijsen, R. Beck, \& R. A. M. Walterbos (Aachen: Shaker), 191

Tilanus, R. P. J., Allen, R. J., van der Hulst, J. M., Crane, P. C., \& Kennicutt, R. C. 1988, ApJ, 330, 667

Torresani, B. 1995, Continuous Wavelet Transform (Paris: Savoire)

Tosa, M. 1973, PASJ, 25, 191

Tosaki, T., Hasegawa, T., Shioya, Y., Kuno, N., \& Matsushita, S. 2002, PASJ, 54, 209

Tully, R. B. 1974, ApJS, 27, 415

Zimmer, P., Rand, R. J., \& McGraw, J. T. 2004, ApJ, 607, 285 